LA GRANJA:

\section{REVISTA DE}

CIENCIAS DE LA VIDA

pISSN:1390-3799; eISSN:1390-8596

http:/ / doi.org/10.17163/lgr.n27.2018.03
Artículo científico / Scientific paper

ECOLOGÍA ACUÁTICA

\title{
PRINCIPIOS FUNDAMENTALES EN TORNO A LA CALIDAD DEL AGUA, EL USO DE BIOINDICADORES ACUÁTICOS Y LA RESTAURACIÓN ECOLÓGICA FLUVIAL EN ECUADOR
}

\author{
MAIN PRINCIPLES ON WATER QUALITY, THE USE OF AQUATIC BIOINDICATORS \\ AND FLUVIAL ECOLOGICAL RESTORATION IN ECUADOR
}

Esteban Terneus-Jácome ${ }^{1}$ y Patricio Yánez ${ }^{1,2}$

${ }^{1}$ Escuelas de Biología Aplicada y de Gestión Turística, Universidad Internacional del Ecuador. Av. Jorge Fernández s/n y Av. Simón Bolívar, Quito-Ecuador.

${ }^{2}$ Instituto de Investigaciones Científicas y Tecnológicas, Universidad Iberoamericana del Ecuador, Av. 9 de Octubre N25-12 y Colón, Quito-Ecuador.

*Autor para correspondencia: hterneus@internacional.edu.ec

\begin{abstract}
Resumen
El presente trabajo se desarrolló como complemento al componente de restauración ecológica contemplado dentro del marco de acción del Plan de Gestión Ambiental del FONAG (Fondo para la conservación del Agua), institución adscrita al Municipio de Quito. El documento forma parte de una línea base de información que gira en torno a la construcción de un escenario adecuado para emprender actividades de restauración ecológica fluvial, en los ambientes en los que ésta fuera necesaria. A nivel municipal es importante que las consideraciones discutidas en la presente investigación así como las iniciativas de restauración ecológica fluvial se incluyan como un componente más dentro de los planes de gestión ambiental que cada gobierno local (GAD) emprende en el territorio de su jurisdicción, y se aborde como un elemento que requiere un permanente seguimiento y monitoreo para detectar oportunamente cambios en la calidad y cantidad del agua que se puedan presentar en una determinada zona y que a la vez esto permita tomar las acciones de manejo que correspondan.

Palabras clave: calidad del agua, Ecuador, bioindicadores, restauración ecológica fluvial.
\end{abstract}




\begin{abstract}
This work was developed as a complement to the ecological restoration component contemplated within the framework of action of the Environmental Management Plan of FONAG (Fund for Water Conservation), an institution attached to the Municipality of Quito. The document is part of the baseline in the creation of a suitable scenario to undertake activities of rivers ecological restoration, where it is necessary. At the municipal level, it is important that the considerations discussed in this paper, as well as initiatives for river restoration, should be inserted as one of the main components within the environmental management plans that each local government (GAD) has to undertake in the territory of its jurisdiction, and it is addressed as an element that requires a permanent monitoring in order to detect the changes in the quality and quantity of water that can be presented in a given area and the corresponding management actions to be taken.
\end{abstract}

Keywords: Water quality, Ecuador, bio-indicators, rivers ecological restoration.

Forma sugerida de citar: Terneus-Jácome, E. y Yánez, P. 2018. Principios fundamentales en torno a la calidad del agua, el uso de bioindicadores acuáticos y la restauración ecológica fluvial en Ecuador. La Granja: Revista de Ciencias de la Vida. Vol. 27(1):36-50. http:/ / doi.org/10.17163/lgr. n27.2018.03. 


\section{Introducción}

\subsection{Diagnóstico de la problemática del agua en Ecuador}

Durante muchos años, en Ecuador el manejo del agua se ha centrado en iniciativas para mejorar el aprovisionamiento de la misma en cantidad, no necesariamente en calidad. A esto suele sumarse la limitada disponibilidad de recursos económicos para la conservación de fuentes primarias de agua y de un criterio de manejo a nivel de la cuenca hidrográfica como unidad de análisis. Por consiguiente, los esfuerzos por prevenir la contaminación y recuperar los cuerpos de agua contaminados han sido mínimos, o su alcance ha respondido a intereses particulares (Solanes y Peña, 2003). En la actualidad, Quito no cuenta con una planta de tratamiento de aguas de alcantarillado, salvo pocas excepciones focalizadas a nivel industrial. Se prevé que para 2018, la ciudad contará con su primera planta de tratamiento de agua (Calles, 2012); hasta tanto las principales cuencas y microcuencas receptoras de las aguas contaminadas provenientes de la ciudad vierten sus aguas a los ríos Machángara, Guayllabamba y Monjas sin ningún tipo de tratamiento. Este último, por ejemplo, recibe las aguas de las diferentes actividades antropogénicas provenientes del páramo del Atacazo y otras zonas cercanas.

A nivel nacional, únicamente la ciudad de Cuenca y algunos sectores de Guayaquil y Loja cuentan con sistemas de tratamiento de aguas de alcantarillado, lo que les ha permitido disminuir las tasas de parasitosis, enfermedades intestinales de las personas y la pérdida de biodiversidad acuática a causa de la contaminación de cuerpos de agua cercanos a centros poblados y/o urbanos (Lloret, 2002).

Las limitaciones de disponibilidad de agua en términos de calidad y el importante crecimiento demográfico nacional están ejerciendo una fuerte presión sobre las zonas altas de páramos de donde se originan las fuentes de agua primarias, generando como consecuencia la sobre explotación del recurso y el deterioro de su cobertura vegetal natural en las áreas de recarga. Por consiguiente, urge tomar medidas de gestión ambiental que permitan recuperar estas fuentes de agua primarias que ya han sido intervenidas, pero que se pueden restaurar con un manejo adecuado y recuperación de las zonas de riberas junto a los cuerpos de agua, y un plan de delimitación de zonas de protección hidrológica (Gon- zález y García, 2007; Magdaleno, 2011; Rodríguez Quiñónez, 2012; Ramírez López, 2015).

\subsection{El marco legal de referencia}

La Carta Magna de la República del Ecuador de 2008, en su capítulo siete (Art. 71), establece los derechos de la naturaleza como primordiales para garantizar el Buen Vivir de las personas en un entorno natural, sano y libre de contaminación. De igual manera, el artículo 72 del mismo capítulo hace referencia a los derechos que tiene la naturaleza a ser restaurada en casos de intervención por parte del ser humano, como resultado de sus actividades socioeconómicas. También señala que, en caso de ocurrir impactos ambientales, los causantes de los mismos están obligados a tomar medidas mitigatorias y restauradoras para devolver el estado natural original al ambiente disturbado (Asamblea Constituyente, 2008).

Para el cumplimiento de lo indicado, el Estado ecuatoriano a través de la máxima autoridad ambiental del país, el Ministerio de Ambiente (MAE), ha generado una serie de leyes, normas y sistemas de control que permiten regular y monitorear las actividades generadas por el ser humano y que atentan o pudieran estar atentando a la integridad de la naturaleza y su funcionalidad ecosistémica.

En su capítulo segundo, la misma Carta Magna hace referencia a la biodiversidad y a los recursos naturales. En el artículo 395 se establece que las políticas de gestión ambiental del Estado deberán ser aplicadas por toda entidad de origen público o privado y serán obligatorias en su cumplimiento. El artículo 400, a su vez, hace referencia a la importancia de conservar la biodiversidad y los recursos naturales de toda persona natural o jurídica, declarándola como una acción de interés público.

La Ley de Gestión Ambiental de Ecuador pone de manifiesto que la máxima autoridad ambiental (Ministerio del Ambiente, 2003) tiene la potestad sancionadora, en caso de comprobarse daños ambientales provocados por actividades procedentes del sector público o privado en cualquier ámbito de acción, así como las atribuciones de otorgar el licenciamiento y permisos de funcionamiento a estas instituciones.

En la sección dos, en el artículo 47 se hace mención a la importancia de las áreas de manejo especial, en las que el FONAG ha considerado a las fuentes de agua primarias (quebradas de páramo) como 
parte de estos ecosistemas por su riqueza única de especies de flora y fauna nativas. En el capítulo III, artículo 57, también se hace referencia a la obligatoriedad de la recuperación y mitigación de áreas degradadas por impactos ambientales negativos.

La Ley de Gestión Ambiental en sus artículos 16 pone de manifiesto la importancia, los principios y directrices de la política ambiental que las instituciones públicas y privadas deben fomentar en cada una de sus actividades. Además, esta ley señala los límites permisibles, los controles y sanciones en materia ambiental. En concordancia con este marco jurídico se encuentra la Ley de Prevención y Control de la Contaminación Ambiental, en la que se hace referencia a ambos procesos en cuanto al aire (capítulo V); las aguas (capítulo VI) y los suelos (capítulo VII), constituyéndose en el marco regulatorio sobre el cual el FONAG enmarca sus procedimientos de gestión ambiental.

Por otra parte, el Ministerio del Ambiente (2003) (Texto Unificado de Legislación Secundaria, Medio Ambiente) es una norma técnica que se ampara en la Ley de Gestión Ambiental y en el Reglamento a la Ley de Gestión Ambiental para la Prevención y Control de la Contaminación Ambiental y se somete a las disposiciones de éstos, es de aplicación obligatoria y rige en todo el territorio nacional.

Esta norma técnica aborda o establece:

a. Los límites permisibles, disposiciones y prohibiciones para las descargas en cuerpos de aguas o sistemas de alcantarillado (Libro VI anexo I).

b. Los criterios de calidad de las aguas para sus distintos usos (Libro VI anexo I); y,

c. Los métodos y procedimientos para determinar la presencia de contaminantes en el agua (Libro VI anexo I).

Todas estas normas y regulaciones deben ser tomadas en cuenta para el funcionamiento y los procesos de gestión ambiental que el FONAG contempla dentro de su planificación estratégica.

El artículo 318 de la misma Constitución consagra al agua como patrimonio nacional estratégico de uso público; y el Estado, a través de la autoridad única del agua SENAGUA (Secretaria Nacional del Agua), creada mediante Decreto Ejecutivo 1088 del 15 de mayo de 2008, genera la propuesta de la nueva Ley de Aguas (Asamblea Nacional, 2010), y a su vez es el responsable de la planificación y gestión de los recursos hídricos que serán administrados bajo este orden de prelación: a. Consumo humano. b. Riego que garantice la soberanía alimentaria. c. Caudal ecológico, y d. Actividades productivas.

Bajo este contexto y estructura legal, el manejo de los recursos hídricos en el Ecuador ha intentado regular y administrar el uso adecuado del recurso. Sin embargo, es perentorio fortalecer el elemento técnico para incrementar la eficacia, eficiencia y operatividad a estas iniciativas (Asamblea Nacional, 2010).

\section{Grupos biológicos indicadores de la calidad del agua}

\subsection{Los macroinvertebrados como bioindi- cadores de calidad ambiental}

La alta presión antrópica a la que han estado expuestos los ecosistemas de agua dulce en Ecuador durante los últimos veinte años pone en evidencia el deterioro de los cuerpos de agua, tanto en cantidad como en calidad. Una de las formas más exitosas y económicas para llegar a determinar el nivel de impacto y el tipo de contaminante o elemento exógeno presente en un cuerpo de agua es utilizando diferentes organismos acuáticos como indicadores de estos cambios o perturbaciones (González y Lozano, 2004; Escobar, Terneus y Yánez, 2013; Terneus, 2015).

Un organismo bioindicador es una especie o grupo de especies que poseen requerimientos ambientales particulares con relación a un conjunto de variables físicas o químicas; esta especie o estas especies pueden presentar cambios en su presencia y distribución espacial, número, morfología o conducta cuando las condiciones del sistema ecológico se alteran (Rosenberg y Resh, 1993). En definitiva, dichos organismos ocupan un hábitat a cuyas exigencias ambientales se encuentran adaptados; cualquier cambio en las condiciones ambientales se reflejará en la estructura, composición y dinámica de las comunidades de macroinvertebrados acuáticos que allí habiten (Terneus, Racines y Hernández, 2012).

Entre estos organismos, los macroinvertebrados como bioindicadores, juegan un papel importante en el manejo adecuado del recurso hídrico.

Dentro de estos requerimientos particulares se ha llegado a determinar que cada grupo o gremio 
de macroinvertebrados acuáticos muestra niveles de especialización o preferencia por ocupar microambientes específicos; entre estos: micro hábitats rocosos, fangosos, de hojarasca, arena, limo o arcillas. A esto se añade la preferencia por ciertos aspectos físicos (hidrodinámicos) como la dinámica del agua y los flujos de corriente: zonas de corriente fuerte, media o débil o la presencia de elementos químicos (Figura 1). Por tanto, la presencia, abundancia, ausencia de estos organismos suelen indicar las condiciones del cuerpo de agua o de un sector de él.

La presencia o no de determinados grupos característicos como los efemerópteros, plecópteros y tricópteros (en la cual se basa el índice EPT) son indicadores de buena calidad de agua, y muchos de ellos ocupan espacios de aguas rápidas, bien oxigenadas y poco profundas, mientras que los dípteros quironómidos y ceratopogónidos y ciertos anélidos ocupan aguas someras, fangosas y profundas por lo que son indicadores de aguas con alta carga orgánica. La relación de estos grupos en proporción y riqueza proporcionan información bastante precisa sobre el estado de salud del ambiente acuático (Jacobsen, Schultz y Encalada, 1997; Giacometti y Bersosa, 2006).

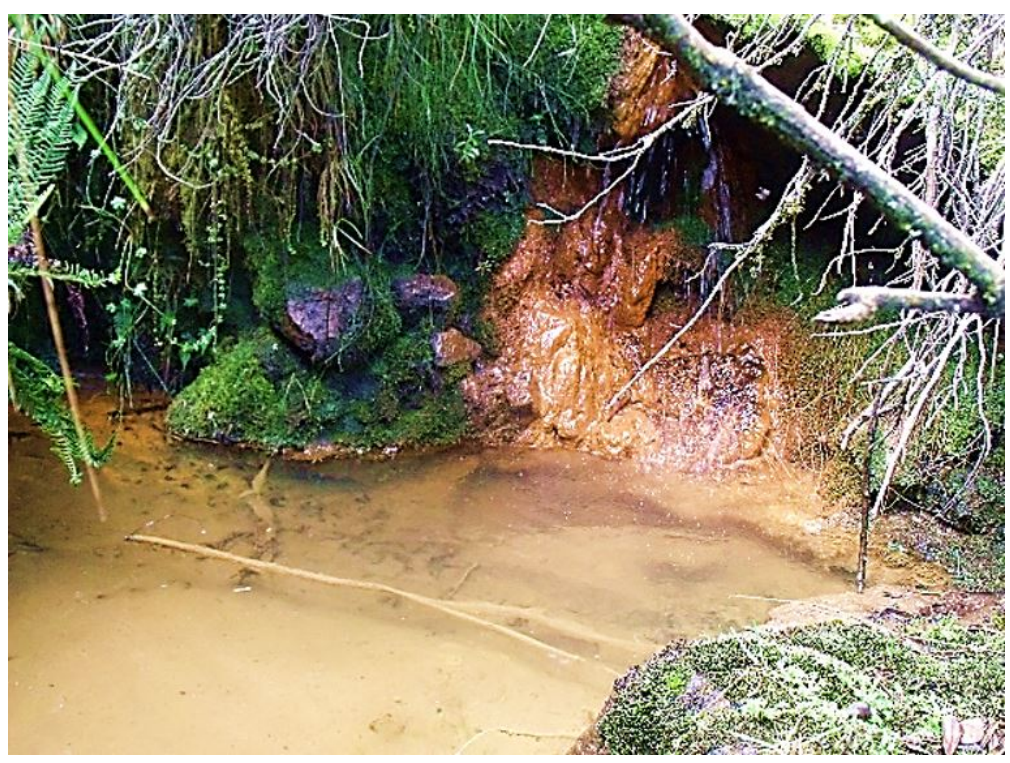

Figura 1. Fuente de agua primaria en un páramo ecuatoriano con alto contenido de hierro. A ella se encuentran adaptadas solo ciertas especies de macroinvertebrados

2.2 Descripción general y características de los principales grupos de macroinvertebrados acuáticos como bioindicadores

A continuación, se destacan algunas características generales de los principales grupos bioindicadores a partir del trabajo de Racines (2014), quien demuestra cómo los macroinvertebrados acuáticos pueden expresar el estado de salud ecológica, a partir del cálculo de índices de tolerancia y sensibilidad a la contaminación del agua en los páramos ecuatorianos.

Los oligoquetos acuáticos (clase Oligochaeta) conforman uno de los grupos más importantes de los invertebrados presentes en lagos, ríos y embalses. Tienen parámetros morfológicos similares a la de oligoquetos terrestres: su tamaño varía entre 1 y $30 \mathrm{~mm}$, se alimentan de algas filamentosas, su respiración es cutánea. Los oligoquetos constituyen un eslabón importante en la cadena trófica bentónica, principalmente en aguas eutrofizadas o contaminadas, en las que alcanzan densidades muy elevadas y sirven de alimento para peces bentónicos, turbelarios, sanguijuelas, nemátodos y larvas de insectos (Brinkhurst, 1980).

Los miembros de este grupo han sido definidos como bioindicadores especiales para diferentes parámetros físicos y químicos, como por ejemplo: ti- 
pos de sustrato, carbono orgánico, fósforo y varios metales pesados, generalmente residuos de hidrocarburos (Chapman, Farrell y Brinkhurst, 1982).

Por otra parte, el orden Coleóptera que presenta la mayor diversidad de especies del mundo, viven en aguas continentales lóticas y lénticas, aguas limpias, con concentraciones de oxígeno alto y temperaturas medias (Roldan Perez, 1988). Poseen alas anteriores o élitros, alas posteriores membranosas, aparato bucal mordedor (Hickman, Roberts y Larson, 2009). Las larvas de los coleópteros presentan formas muy diversas, el abdomen presenta agallas laterales o ventrales, tienen metamorfosis completa y el adulto es morfológicamente muy diferente (Figura 2).

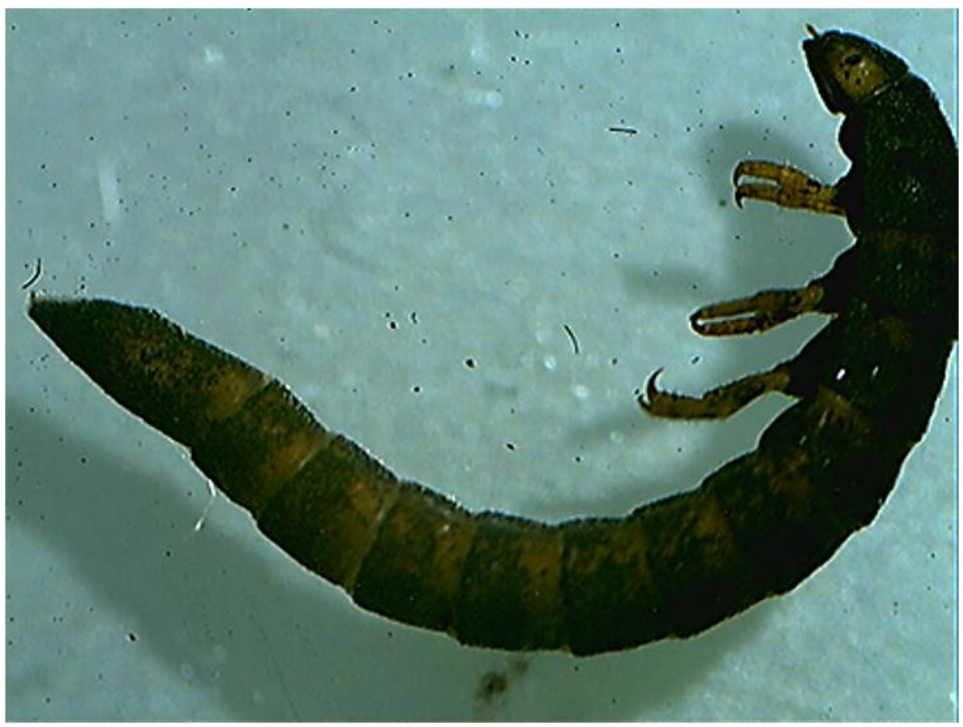

Figura 2. Larva de un coleóptero de la familia Elmidae. Junto a sus patas se encuentran las agallas que le permiten respirar en medios acuáticos con alta concentración de oxígeno.

El orden Díptera es el más complejo, abundante y mejor distribuido en el Planeta. Está conformado por insectos holometábolos, es decir, sus ciclos de vida consisten en huevos, larvas acuáticas, pupas y adultos voladores, de los cuales la fase bioindicadora es la larva. Su hábitat lo conforman ríos de aguas estancadas y corrientes (Roldan Perez, 1996). Las características más importantes de las larvas de los dípteros son la ausencia de patas torácicas, el cuerpo blando y cubierto de cerdas, espinas apicales o corona de ganchos en prolongaciones que ayudan a la locomoción y adhesión al sustrato (Roldan Perez, 1988).

Los Dípteros son considerados los mejores indicadores de la presencia de un alto grado de materia orgánica en los cuerpos de agua, sus familias más frecuentes son Psychodidae, Tipulidae, Blephariceridae, Culicidae, Ceratopogonidae, Chironomidae, Simuliidae, Tabanidae y Muscidae (Roldan Perez, 1996). A la familia Chironomidae se la asocia con aguas de abun- dante presencia de materia orgánica (en sistemas lóticos y lénticos), y bajas concentraciones de oxígeno disuelto. Sin embargo, dentro de esta familia existen unos pocos géneros que se desarrollan mejor en aguas limpias con altas concentraciones de oxígeno (González y Lozano, 2004). Las larvas de la familia Tipulidae son comunes en los sedimentos o entre las hojas del fondo de corrientes o escurrideros, troncos podridos y otra materia vegetal en descomposición (Lanza, Hernández y Carbajal, 2000).

Los chinches verdaderos (orden hemípera) poseen un tamaño de 2 a $100 \mathrm{~mm}$ con alas o sin ellas, alas posteriores membranosas, aparato bucal perforador-chupador. En este orden se incluyen a los escorpiones de agua, zapateros, chinches de cama, de campo, triatomas, pentatomas (Hickman, Roberts y Larson, 2009). El suborden Heteróptera comprende importantes insectos acuáticos que habitan una amplia gama de ecosistemas acuáticos (de agua dulce, ambientes marinos e intersticiales y de 
altura). La mayoría son depredadores y en algunos casos detritívoros y alguívoros como los de la familia Corixidae (Konopko y Melo, 2009). Los hemípteros acuáticos incrementan su abundancia en sistemas con profundidades menores a $1 \mathrm{~m}$. El oxígeno disuelto en el agua permite que estos organismos permanezcan más tiempo sumergidos, ya que su burbuja de aire utilizada para la respiración dura más tiempo (Contreras, Navarrete y Lara, 2008); también poseen otras adaptaciones para respirar tomando oxígeno del aire como tubos anales y canales abdominales (Roldan Perez, 1988). La presencia de vegetación acuática sumergida disminuye la depredación de los individuos de la familia Corixidae (Contreras, Navarrete y Lara, 2008). Los individuos de la familia Naucoridae son enteramente acuáticos y se encuentran generalmente en sistemas lóticos y lénticos, entre hidrófitas vasculares (Lanza, Hernández y Carbajal, 2000).

Los individuos de la clase Gasterópoda, mayoritariamente herbívoros (Hickman, Roberts y Larson, 2009), se alimentan de algas y varios residuos vegetales. Habitan ambientes con sales disueltas, especialmente carbonato de calcio, siendo indicadores de aguas alcalinas. La mayor parte de especies necesitan altas concentraciones de oxígeno (Roldan Perez, 1988).

Los individuos del orden Tricladida (planarias y especies de cuerpo plano) pueden alcanzar 30 $\mathrm{mm}$ de longitud. Son fundamentalmente carnívoras, la mayoría viven bajo piedras, hojas, ramas y en aguas poco profundas; sus individuos requieren aguas oxigenadas. Sin embargo, algunas tienen la capacidad de soportar niveles de contaminación (Roldan Perez, 1988).

Los individuos de la clase Nematomorpha reciben el nombre de "gusanos crin de caballo", los gusanos adultos miden entre 10 y $70 \mathrm{~cm}$ de longitud, poseen una cutícula fibrosa; viven en corrientes limpias, adheridos a la vegetación, bajo piedras, a orilla de ríos y arroyos (Roldan Perez, 1988). Son organismos que necesitan completar su ciclo de vida dentro de un huésped apropiado.

Dentro de la Clase Hirudinea, los individuos del orden Glossiphoniforme, llamados sanguijuelas, tienen tamaños de entre $5 \mathrm{~mm}$ a $45 \mathrm{~cm}$, su cuerpo es plano, poseen ventosas que rodean la boca (Hickman, Roberts y Larson, 2009). Algunas se alimentan de residuos orgánicos, pero la mayoría son carnívoras. Realizan intercambio gaseoso a través de la piel la cual tiene una innumerable cantidad de capilares
(Roldan Perez, 1996).

El orden Acari (ácaros), perteneciente a la Clase Arachnoidea, es un grupo poco conocido, sus individuos tienen forma globular, con cefalotórax y abdomen fusionados, su tamaño varía entre 0,4 y 3 mm (Hickman, Roberts y Larson, 2009). Los ácaros acuáticos se encuentran en la mayoría de hábitats dulceacuícolas, tanto en ambientes lénticos como lóticos.

Los individuos de la Clase Bivalva varían entre 2 y $180 \mathrm{~mm}$ de largo, son filtradores de plancton y detritus. Los bivalvos de agua dulce son organismos tanto de aguas lóticas como lénticas. Se los encuentra en ecosistemas fangosos, son abundantes donde el $\mathrm{pH}$ del agua está por encima de 7,0 y donde existe gran cantidad de carbonatos.

\subsection{La variabilidad en la composición y estructura de los macroinvertebrados acuáticos}

Las comunidades de macroinvertebrados bentónicos han sido seleccionadas como uno de los grupos de organismos más relevantes para valorar la integridad del estado ecológico de los cuerpos de agua, ya que han demostrado ser buenos bioindicadores de la calidad del medio al cumplir con algunos requisitos deseados como sensibilidad, factibilidad de muestreo, validez científica, entre otros (González y Lozano, 2004).

Sin embargo, y a pesar de las innumerables ventajas de estos organismos, se ha detectado que la estacionalidad puede ser un factor determinante que influye sobre la presencia de ciertos grupos o especies, y sobre la importancia de la determinación de la abundancia y diversidad de macroinvertebrados en relación con el clima; esto deja en claro que la estructura y composición de macroinvertebrados no varía solamente a causa de impactos ambientales antrópicos que afectan la calidad del agua, si no también muestran variaciones importantes como consecuencia de la variabilidad natural de las condiciones ambientales en los ecosistemas acuáticos (Chang et al., 2014).

En ocasiones, esta variabilidad natural (intraanual o estacional e interanual) que presentan las comunidades de macroinvertebrados acuáticos no permite utilizar el mismo criterio para evaluar las comunidades presentes en dos tramos diferentes de un cuerpo de agua, así como en dos épocas distintas, generando un cierto grado de incertidumbre en 
los procesos de evaluación de la calidad del medio (Loeb y Spacie, 1994).

Las variaciones intra-anuales suelen seguir patrones cíclicos y direccionales, los cuales son relativamente predecibles, ya que están principalmente determinados por la variación estacional que sigue el clima. Sin embargo, las segundas (interanuales) son menos predecibles debido a que suelen derivar de fenómenos generados a una escala mayor, los cuales no siguen un patrón cíclico tan definido (Reynoldson y Wright, 2000).

La variación temporal de las comunidades de macroinvertebrados puede interferir en el proceso de evaluación del estado ecológico de los cuerpos de agua, incluso en el ámbito de dar resultados comparativos. Algunos autores, que han abordado este problema, proponen ciertos puntos a tomar en cuenta para disminuir los efectos que la variabilidad temporal pudiera causar sobre las comunidades de macroinvertebrados acuáticos, en tales consideraciones el clima suele ser el principal factor de determinación de variantes.

Por lo que se pone de manifiesto la ventaja de caracterizar la comunidad de macroinvertebrados al menos en dos épocas del año con diferentes condiciones ambientales, esto suele permitir el conocer cómo responde la comunidad frente a un rango de valores de variables ambientales, y así realizar las comparaciones necesarias para determinar que su presencia es por causa de contaminación y no por la estacionalidad. Sporka et al. (2006) manifiesta las ventajas de trabajar con bases de datos que contengan información de varias épocas dentro de un mismo año, ya que las variaciones climáticas de los últimos tiempos pueden ser las claves en tener un margen de error al momento de comparar estudios realizados anteriormente.

En estos casos, la toma de datos de campo se debe realizar siempre en la misma época del año, para que la comparación entre muestras sea suficientemente efectiva. Para ello, es necesario identificar cuál es la época del año en la que los cambios generados por las presiones antrópicas en las comunidades de macroinvertebrados son más notables y por lo tanto más fáciles de identificar; en este caso habría que colocar los monitoreos preferiblemente en los momentos en que los efectos de estas presiones sean mayores.

\section{La gestión ambiental y los proce- sos de restauración ecológica flu- vial}

\section{1 ¿Qué es la restauración ecológica flu- vial?}

La gestión ambiental está concebida como el sistema integrado de procesos y acciones destinadas a conseguir la recuperación ambiental de un área o zona degradada a partir de un análisis diagnóstico y una planificación. Dentro de este marco, la restauración ecológica fluvial representa un componente importante del proceso de gestión ambiental, el cual está encaminado a conseguir la recuperación del estado funcional de un cuerpo de agua hasta alcanzar su funcionalidad ecosistémica. Restaurar consiste en recuperar un sistema fluvial natural eliminando aquellos impactos o alteraciones que lo degradan, permitiendo así que se restauren los procesos y equilibrios naturales que facilitan a su vez que dicho sistema funcione de forma auto-sostenida en el tiempo (Herrera, 2013). Sin embargo, este proceso de restauración no siempre permite alcanzar las condiciones originales del sistema, transformándolo de todas maneras en un río de dinámica antropocéntrica, en lugar de la dinámica ecocéntrica propia de cuerpos de agua en estados prístinos u originales.

Para que un río recupere sus funciones naturales deberá recuperar su caudal y el espacio fluvial en el tiempo, lo que en la práctica es imposible, pero si es factible conseguir unos parámetros de aproximación lo más cercanos posibles al comportamiento histórico del río. Esto se lo puede conseguir satisfactoriamente en la medida en que se reduzca el número de impactos ambientales negativos ocasionados sobre el cuerpo de agua.

Un elemento importante en el proceso de restauración es la participación de la comunidad, donde los usuarios del recurso deberán preguntarse: ¿Qué río queremos? y según esto, los actores locales van definiendo alcances de intervención, que a su vez determinan el grado de recuperación del cuerpo de agua. Este proceso debe ir acompañado de un fuerte componente de sensibilización y educación ambiental hacia la comunidad que se beneficia del recurso en una determinada zona (Herrera, 2013). 


\subsection{Objetivos de la restauración ecológica fluvial}

El objetivo general de la restauración ecológica fluvial es recuperar la funcionalidad ecosistémica de uno o más cuerpos de agua, tanto en sus aspectos ecológicos como en los hidrogeológicos, de tal manera que estos cuerpos recuperen su condición de prestadores de servicios ecosistémicos hacia el ambiente y sus diferentes componentes.

Para conseguir este objetivo, es necesario identificar los siguientes aspectos que influyen directamente sobre la calidad ecológica e hidrogeológica de los cuerpos de agua: dinámica fluvial, biodiversidad local, resiliencia, compatibilidad con el entorno socio económico y ambiental, entorno paisajístico, valores patrimoniales y efectivos, legislación, presencia y tipología de las inundaciones y relación con el empleo en la zona (Herrera, 2013).

La dinámica fluvial representa la capacidad de un cuerpo de agua para marcar su trayectoria de acuerdo a un flujo de caudales y dinámica energética de sus aguas, en función de la pendiente y del relieve por donde surcan sus aguas. Esta dinámica de expresión física desempeña un papel fundamental en el ciclo de nutrientes del sistema, en la composición biológica del mismo y en la capacidad de erosión del río por la fuerza de choque de sus aguas. Toda esta dinámica se traduce, a la final, en la capacidad de autodepuración del río frente a los disturbios naturales o provocados que pueda enfrentar el cuerpo de agua (Elliot Munro, 2010).

Lo anterior va enlazado al tema de biodiversidad, que también es un aspecto muy importante porque permite medir y evaluar el estado de salud ecológica de un ecosistema y refleja claramente los efectos ocasionados por actividades de intervención. Es necesario considerar además que no todas las especies que pueden estar conformando la biota de un río son beneficiosas para el ambiente acuático. Existen especies exóticas o introducidas que pueden afectar seriamente la salud ecológica del ecosistema, es el caso de algunas especies de peces y plantas acuáticas, las mismas que en ocasiones atentan contra la salud poblacional de otras especies homólogas o en su defecto, pueden transformarse en plagas invasivas de difícil control y que demandan una atención inmediata de manejo (Fernández, Leguizamon y Acciareci, 2015).

La recuperación de la (auto) funcionalidad ecosistémica de un cuerpo de agua se la conoce como resiliencia, esta condición permite conocer en qué capacidad se encuentra un cuerpo de agua para soportar niveles de intervención antrópica o factores de cambio de las condiciones naturales y auto depurarse. Este fenómeno también está vinculado estrechamente con los procesos o iniciativas de restauración ecológica fluvial (Ferreira, 2012).

Todo proceso de restauración fluvial debe tomar en cuenta el contexto socio-económico y paisajístico en el que se desarrolla, ya que de los intereses de las personas asociadas a la dinámica del río dependen los niveles de intervención recibidos por el cuerpo de agua y las consideraciones de manejo que se den sobre el mismo. Por ejemplo, un entorno paisajístico dominado por actividad agrícola y ganadera, no tendrá el mismo impacto ambiental que un rio que está rodeado por un poblado donde predominan las edificaciones. Además, los intereses de conservación del primero se fundamentan en garantizar el aprovisionamiento de agua en calidad y cantidad, debido a que la comunidad utiliza el recurso hídrico como fuente de riego y abrevadero para los animales. En el segundo caso quizá no sea muy importante cuidar el estado de salud ecológica del río porque seguramente disponen de agua potable y lo utilizan en su lugar como sumidero de desechos; por consiguiente, los propósitos y acciones de restauración tienen distintos alcances, dependiendo de los intereses socio-económicos de la población circundante.

En algunos contextos, la presencia de un río como parte de su entorno paisajístico representa un elemento de riqueza etnográfica y cultural que, por tradición, merece un trato especial. En estos casos, el río se transforma en una fuente de rituales y costumbres culturales (sitios de ocio, turismo, relajación, recarga espiritual, etc.) cuyas consideraciones habrá también que tomar en cuenta al momento de restaurarlo.

Es necesario también observar la legislación (nacional, provincial, municipal) de cada sitio o área a restaurar para determinar el alcance y viabilidad de la misma. Cada estado o provincia tienen su propio marco legislativo que regula este tipo de actividades según la normativa de cada país (Asamblea Nacional, 2010). Para el Ecuador, la ley de recursos hídricos es el instrumento que permite regular el uso y manejo del recurso hídrico a nivel nacional, bajo la rectoría única de la Secretaría Nacional del Agua (SENAGUA) y la coordinación con el Ministerio del Ambiente (MAE) como autoridad ambiental nacio- 
nal.

Por último, un proceso de restauración demanda situaciones de intervención eventualmente complejas y en muchas ocasiones multidisciplinarias, que requieren de cierto contingente de mano de obra. Por tanto, los procesos de restauración pueden generar fuentes de empleo, en los que las comunidades aledañas a los lugares de intervención pueden ser participantes activos dentro de la ingeniería del proceso de restauración fluvial (Figura 3).

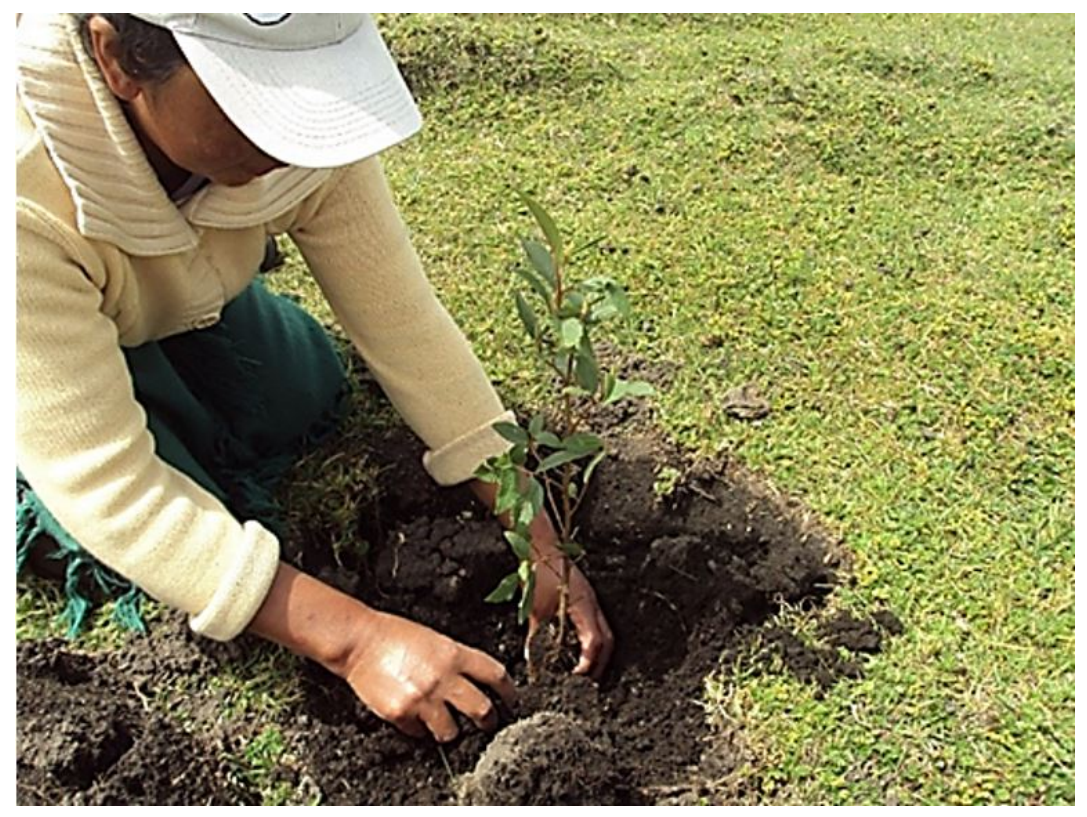

Figura 3. Persona de la comunidad Espejo, páramo del Atacazo, participando en un proceso de restauración ecológica (reforestación con especies nativas) en las riberas de cuerpos de agua seleccionados.

\subsection{Algunas estrategias para restaurar un ambiente fluvial}

Para recuperar un espacio fluvial es necesario referirse a información histórica del área afectada o a ser intervenida. Esto permitirá tener un punto de referencia sobre el cual se medirán, a mediano y largo plazo, la eficiencia de las acciones tomadas en términos de restauración. Si no es factible conseguir esta información histórica, habrá que hacer un análisis de las condiciones de referencia de cuerpos de agua próximos o aledaños a la zona de intervención.

Uno de los aspectos más importantes es instau- rar un régimen natural de caudales con el objetivo de no dilapidar ingentes esfuerzos económicos y humanos sobre una zona o tramo de río en el que no se conoce el comportamiento y dinámica de crecidas. De este conocimiento depende la sobrevivencia o resguardo de las especies u obras complementarias que se hagan en el área de restauración.

La eliminación de barreras u obstáculos dentro del cauce del canal fluvial (Figura 4) es otro de los aspectos a considerar debido a la importancia para mantener la conectividad hídrica del cauce y de esta manera, favorecer la migración de especies y el intercambio genético de las mismas. 


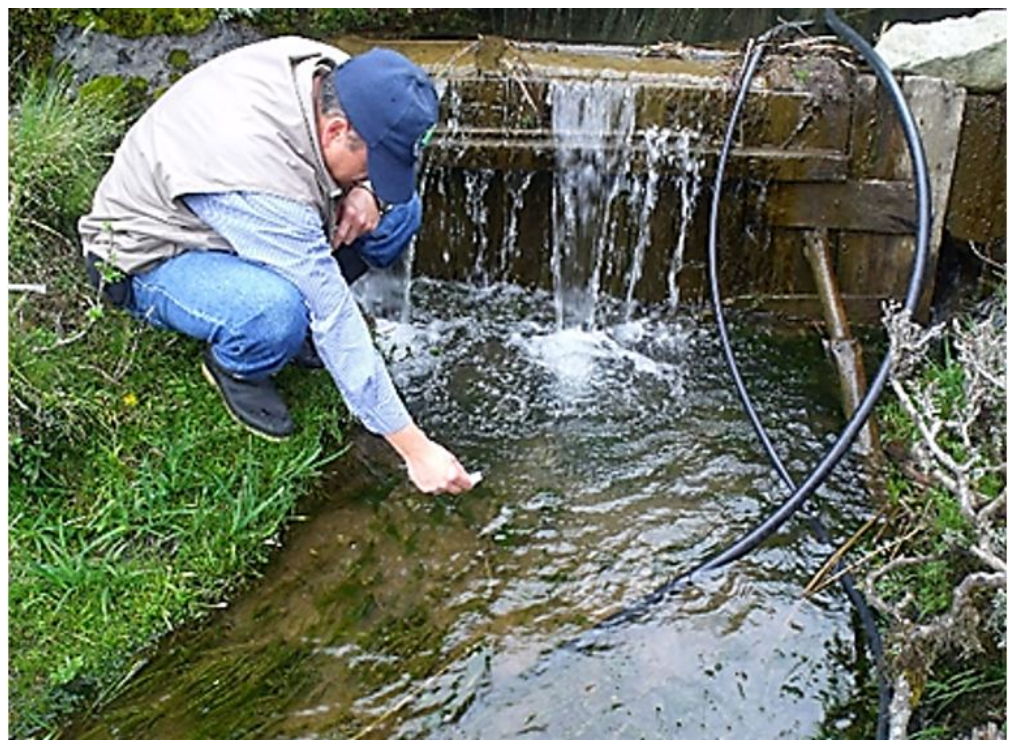

Figura 4. Presencia de una barrera artificial colocada en el lecho de un río de páramo (muro de represamiento).

El establecimiento de zonas naturales de protección hidrológica en forma de franjas paralelas a los costados de los cauces de agua suelen ayudar a retener sedimentos que de otra manera pudieran caer al cuerpo de agua, ya que la vegetación circundante actúa como filtro natural, reteniendo sólidos que pudieran transformarse en causas de serios procesos de sedimentación de los cuerpos de agua. Estas zonas de protección hidrológica deberían estar estructuradas por vegetación natural heterogénea para que cada una de las especies vegetales cumpla un papel específico en el proceso de retención de sólidos (Garcia de Jalón, 2003).

En ocasiones y debido al daño ocasionado en un determinado tramo de río, es necesario acudir a plantaciones seminaturales con especies propias del sector (Figura 3). Este procedimiento es recomendable cuando se requiere acelerar el proceso de restauración frente al tiempo prolongado que tomaría un proceso de regeneración natural espontánea del medio afectado. Normalmente, este tipo de procedimientos demanda un seguimiento prolongado de cuidado para asegurar el adecuado establecimiento de las especies utilizadas para restaurar el medio acuático.

Un aspecto importante que se vincula con el anterior es la creación y regeneración de micro hábitats con el objetivo de recuperar la biota natural del lugar. Esto se fundamenta en la creación de microambientes en los que se genere hojarasca, la misma que incrementará el aumento de carga orgánica en el tramo del río y la formación de limo, condiciones que favorecen el establecimiento espontáneo de algunas especies debido a la disponibilidad de nutrientes. Este escenario puede ser beneficioso para la creación de zonas de freza y refugio para especies mayores como los peces.

En ocasiones es necesario recuperar la biodiversidad del lugar alterado, para lo cual es válido introducir nuevamente especies vegetales preferentemente nativas y con dinámicas demostradas de tolerancia a factores contaminantes (Yánez y Bárcenas, 2012), generando así beneficios socio ambientales. En algunos casos este escenario puede tornarse beneficioso para las actividades productivas de la zona, ya que las comunidades pueden usar el recurso de manera directa o indirecta como mecanismo de subsistencia.

\subsection{La bioingeniería aplicada a la restaura- ción fluvial}

La bioingeniería aplicada a la restauración y regeneración ambiental de los ríos consiste en el uso de plantas vivas o partes de éstas, conjuntamente con otros materiales biodegradables (maderas, rocas, mantas, redes orgánicas etc.) y otros sintéticos, generalmente fotodegradables (geotextiles, redes y geomallas de polipropileno), incorporando y aprovechando los elementos locales (topografía del sue- 
lo, microclima etc.) para conseguir objetivos estructurales en un proceso de restauración fluvial (Herrera, 2013). A partir de esta conceptualización, algunos autores conciben ciertas variaciones dependiendo del resultado final del proceso de restauración. Se conoce como Ecoingeniería el propósito de restaurar que persigue mejorar la funcionalidad ecosistémica dejando fuera el uso de estructuras o insumos artificiales. Otros autores denominan a este proceso como ingeniería naturalística.

Este tipo de técnicas se utilizan para acelerar los procesos de recuperación ecológica de un cuerpo de agua, proceso al que a la naturaleza le tomaría mucho más tiempo recuperarla. La ingeniería convencional se diferencia porque prima el criterio de la funcionalidad de la infraestructura física, en lugar de priorizar la funcionalidad ecosistémica.

Dentro de las técnicas de ingeniería naturalística las que más sobresalen son aquellas que combinan las plantas vivas con material inerte como arena, palos, troncos, piedras y tierra. Con este material se consigue establecer plantaciones, estaquillados, estoconados o trasplantes de rizomas, trazados vivos, enramado y enrejado vivo.

Por otro lado, y como parte de las técnicas de bioingeniería, se puede utilizar material sintético, mezclado con material vivo. Las formas más comunes son: aplicación de mallas plásticas, geomembranas, rollos de fibra de coco, gaviones combinados con biorrollos, etc.

\subsection{Estrategias de gestión para la aplica- ción de la bioingeniería}

En una intervención de restauración hay que tener presente siempre el principio de cautela para no subestimar el potencial hidráulico de choque del río y a su vez, la capacidad de regeneración de la vegetación natural utilizada para la restauración. Hay plantas que crecen más rápido y se establecen más fácilmente que otras, pero no todas van al mismo ritmo. Esto supone un proceso de siembra progresiva y por tramos cortos, para ir evaluando el resultado de prendimiento y adaptación de las nuevas plantas.

Antes de plantear las acciones de restauración es necesario conocer perfectamente la dinámicas fluvial, hidrogeológica y biológica del cuerpo de agua para brindarle la mejor y más cercana aproximación a sus condiciones de origen (Ollero, 2011).

También es importante, luego de recopilar la in- formación (datos históricos, cartográficos, etc.) de las condiciones del sitio a restaurar, efectuar un análisis in situ para verificar que lo planeado en papel realmente será adaptado efectivamente a las condiciones del sitio en particular.

Al final del proceso, es fundamental el mantenimiento y sostenibilidad de las actuaciones, sobre todo durante los primeros tres a seis meses, tiempo en el cual la mayoría de especies vegetales sembradas en sectores contiguos al río se establecen y consiguen adaptarse a las condiciones del medio. Luego de este tiempo se recomienda estructurar un programa de monitoreo y seguimiento que incluya al menos una intervención anual y con el presupuesto adecuado equivalente al $20 \%$ del valor total de la restauración (Herrera, 2013).

\subsection{La participación ciudadana en el pro- ceso de restauración ecológica fluvial}

La única garantía para asegurar la sostenibilidad de las intervenciones de restauración fluvial es la participación ciudadana. Al hacer partícipes a los habitantes de la zona se consigue un empoderamiento de la iniciativa por parte de los actores locales frecuentemente interesados en mantener una buena calidad de los cuerpos de agua para diferentes fines (Nasimba, Yánez y Barros, 2017). Para conseguirlo, el proyecto debe considerar la participación activa de miembros de la comunidad, ya sea en la fase de planificación o en las distintas fases de su misma ejecución (Ollero, 2011).

La fase de socialización de la iniciativa es el aspecto más importante. De esta manera, la comunidad toma conciencia de la importancia de las acciones a tomar y cómo éstas benefician al entorno paisajístico de la zona y, en definitiva, contribuyen a mejorar la calidad de vida de la población en las áreas de influencia de los cuerpos de agua.

En este aspecto la iniciativa de los gobiernos locales con sus distintas figuras de gestión a nivel local tiene a cargo competencias específicas sobre estos temas. Los recursos económicos destinados para estas actividades deben provenir de una partida presupuestaria de los municipios y el sector privado, que garantice la disponibilidad permanente de recursos para asegurar la sostenibilidad de la iniciativa. Los equipos de trabajo deben ser multidisciplinarios y pueden provenir tanto del sector público como del privado. Luego de la fase de intervención del proyecto es recomendable designar comisiones 
de veeduría ciudadana y de gestión para que estas asociaciones sean las encargadas de velar por el adecuado cumplimiento de las actividades previstas y aboguen por la continuidad y sostenibilidad de la iniciativa, valorable en dependencia de la aplicación de estrategias pluriparticipativas pero orientadas a la fiscalización e implementación de medidas judiciales vs. actores contaminantes.

\section{Conclusiones}

La presente investigación ha permitido generar un marco teórico de referencia que busca aportar a las iniciativas de restauración fluvial en Ecuador especialmente, pero no exclusivamente, en ambientes andinos. Esta primera fase del estudio ha permitido confrontar información generada a partir de los impactos ocasionados por la actividad antropogénica y sus posibles consecuencias sobre el estado de salud ecológica de las fuentes de agua primarias, utilizando como termómetros de calidad ambiental al estado de salud de la cobertura vegetal y el recurso hídrico en términos de calidad y cantidad, con el apoyo de bioindicadores acuáticos y variables ambientales físico-químicas básicas.

Es importante señalar que esta investigación constituye una primera fase de un programa institucional de restauración ecológica de fuentes de agua primarias, liderados por el FONAG y en la que la microcuenca del Atacazo ha sido seleccionada como una unidad de análisis piloto a escala local, luego de lo cual sus resultados y experiencias favorables se replicarán a otras microcuencas aledañas a la ciudad de Quito. Los resultados de la fase posterior están siendo sistematizados para ser presentados en próximas publicaciones.

Si bien es cierto se tienen resultados satisfactorios en esta primera fase del proyecto, hay que tener claro que las iniciativas de restauración ecológica fluvial deben arrojar resultados favorables a mediano y largo plazos y para alcanzarlos habrá que conseguir el empoderamiento de la iniciativa por parte de la gente local, como una estrategia para garantizar continuidad de la iniciativa en el tiempo.

Se debe recordar que resulta importante manejar de mejor manera las obras de captación de agua en las zonas altas, con la finalidad de causar el menor daño posible al ecosistema y a las especies asociadas, evitando el corte o interrupción de la conectividad hidrológica del sistema.
A nivel municipal es importante que este tipo de iniciativas de restauración ecológica fluvial se incluyan como un componente más dentro de los planes de gestión ambiental que cada gobierno local (GAD) tenga que emprender en el territorio de su jurisdicción, y se lo aborde como una iniciativa que requiere un permanente seguimiento y monitoreo para detectar oportunamente los cambios que pueden presentarse en una determinada zona y tomar oportunamente las acciones de manejo que correspondan.

\section{Agradecimientos}

A la Universidad Internacional del Ecuador por financiar la mayor parte de la investigación, al igual que el apoyo logístico y de información brindado por el FONAG (Fondo para la Protección del Agua), institución adscrita al Municipio de la ciudad de Quito, y que a través de sus directivos, Dr. Bert De Biévre e Ing. Gustavo Galindo, nos abrieron las puertas y permitieron participar en el proyecto.

Un agradecimiento especial a todas las personas que apoyaron en algún momento el desarrollo de esta investigación, de manera particular a la bióloga María José Racines, por su apoyo y colaboración en el componente de determinación taxonómica de macroinvertebrados acuáticos, al igual que al Ing. Yandry Jumbo, quien asistió en las jornadas de campo y compartió valiosa información del proyecto como técnico del FONAG.

\section{Referencias}

Asamblea Constituyente. 2008. "Constitución de la República del Ecuador." [en línea] disponible en: https://goo.gl/kfu4Qq.

Asamblea Nacional. 2010. “Ley Orgánica de Recursos Hídricos." Registro Oficial 305, Ecuador. [en línea] disponible en: https://goo.gl/VR4c71.

Brinkhurst, R. O. 1980. Pollution Biology - The North American Experience. In Brinkhurst R.O., Cook D.G. (eds) Aquatic Oligochaete Biology. Springer, Boston, MA. [en línea] doi: https://doi.org/10. 1007/978-1-4613-3048-6_24.

Calles, J. 2012. “La contaminación del agua en Ecuador." El agua en el Ecuador. [en línea] disponible en: https://goo.gl/WERwSG. 
Chang, FH, J. E Lawrence, B Rios-Touma y V. H. Resh. 2014. "Tolerance Values of Benthic Macroinvertebrates for Stream Biomonitoring: Assessment of Assumptions Underlying Scoring Systems Worldwide." Environmental Monitoring and Assessment 186(4):2135-2149. [en línea] doi: https: //doi.org/10.1007/s10661--013--3523--6.

Chapman, P, M Farrell y R. Brinkhurst. 1982. “Relative tolerances of selected aquatic oligochaetes to individual pollutant and environmental factors." Aquatic Toxicology 2(1):47-67. [en línea] doi: https: //doi.org/10.1016/0166--445X(82)90005--4.

Contreras, G, C Navarrete y J. Lara. 2008. “Hemípteros Acuáticos en dos estanques piscícolas del estado de México." Revista Chapingo 14(1):39-43. [en línea] disponible en: https://goo.gl/BmC9gS.

Elliot Munro, S. 2010. El río y la forma: introducción a la geomorfología fluvial. RIL Editores. [en línea] disponible en: https:/ /goo.gl/yYNXg1.

Escobar, M. J, E Terneus y P. Yánez. 2013. “El Plancton como bioindicador de la calidad del agua en zonas agrícolas andinas: análisis de caso." Revista Qualitas 5:17-37. [en línea] disponible en: https: //goo.gl/FJs8BB.

Fernández, L, E Leguizamon y H. Acciareci. 2015. Malezas e invasoras de la Argentina. Tomo I: Ecología y manejo. Bahía Blanca: Editorial EDIUNS. [en línea] disponible en: https://goo.gl/UWp1c9.

Ferreira, M. T. 2012. Restauración, rehabilitación y gestión fluvial. Restauración y gestión ecológica fluvial. [en línea] disponible en: https://goo.gl/ HnsNxn chapter 1. Introducción, pp. 15-20.

Garcia de Jalón, D. 2003. Restauración de riberas. In Asociación Española de Ecología Terrestre. Universidad de Alcalá.

Giacometti, J y F. Bersosa. 2006. “Macroinvertebrados acuáticos y su importancia como bioindicadores de calidad del agua en el río Alambi." Boletín Técnico Serie Zoológica 2:17-32. [en línea] disponible en: https://goo.gl/tdZ9mY.

González, L y L. Lozano. 2004. “Bioindicadores como herramientas de evaluación de la calidad ambiental en la parte alta de la Microcuenca las Delicias." Umbral Científico (5):73-82. [en línea] disponible en: https://goo.gl/4JdS87.
González, T y J. García. 2007. Restauración de ríos: guía metodológica para la elaboración de proyectos. In ETSIM Universidad Politécnica de Madrid. [en línea] disponible en: https://goo.gl/Nd8aCP.

Herrera, T. 2013. Manual metodoógico de actuaciones de restauración ambiental y uso público en ámbitos fluviales. Servicio de Ingeniería, Sanidad y Calidad Ambiental, Málaga. [en línea] disponible en: https://goo.gl/dpx5DA.

Hickman, C, L Roberts y A. Larson. 2009. Principios integrales de Zoología. McGraw-Hill / Interamericana de España. [en línea] disponible en: https://goo.gl/J3c5Yp.

Jacobsen, D, R Schultz y A. Encalada. 1997. "Structure and diversity of stream invertebrate assemblages: the influence of temperature with altitude and latitude." Freshwater Biology 38(2):247261. [en línea] disponible en: http://dx.doi.org/ 10.1046/j.1365--2427.1997.00210.x.

Konopko, S y M. Melo. 2009. "Larval morphology of Ectemnostega (Ectemnostegella) montana (Lundblad 1928) (Hemiptera: Heteroptera: Corixidae: Corixinae), with an emphasis on chaetotaxy." Zootaxa 2315:1-18. [en línea] disponible en: https://goo.gl/B1bUvv.

Lanza, G, S Hernández y J. Carbajal. 2000. Organismos indicadores de la calidad del agua y de la contaminación (bioindicadores). Ilustrada, Plaza y Vlades, México. [en línea] disponible en: https: //goo.gl/cgxt6d.

Lloret, P. 2002. Problemática de los recursos hídricos en Ecuador, sistemas y tendencias en el manejo de cuencas hidrográficas. In Quito: Foro de los Recursos Hídricos del Ecuador.

Loeb, S y A. Spacie. 1994. Biological Monitoring of Aquatic Systems. Londres: Lewis Publishers. [en línea] disponible en: https://goo.gl/8SjvE3.

Magdaleno, F. 2011. "Gestión y restauración de los bosques de ribera." Boletín del observatorio de la diversidad biológica y los procesos ecológicos en el medio rural 3:7-14. [en línea] disponible en: https: //goo.gl/1Z5JBs.

Ministerio del Ambiente. 2003. “Texto Unificado de Legislación Secundaria del Medio Ambiente. TULSMA." Ecuador. [en línea] disponible en: https://goo.gl/C75DWr. 
Nasimba, C, P Yánez y L. Barros. 2017. “El agua como componente fundamental en atractivos turísticos naturales: el caso de Las Siete Cascadas de El Zapanal, Pangua, Ecuador." Qualitas 14:67-86. [en línea] disponible en: https://goo. $\mathrm{gl} / \mathrm{pYYRX} 2$.

Ollero, A. 2011. "Restauración fluvial, principios, dificultades y propuestas. La perspectiva del CIREF." Sauce pp. 36-45. [en línea] disponible en: https://goo.gl/h48pe5.

Racines, M. 2014. Aplicación y factibilidad del índice ABI (índice biótico andino) en las principales microcuencas del Parque Nacional Cayambe Coca. In Trabajo de titulación, Escuela de Biología Aplicada, Universidad Internacional del Ecuador.

Ramírez López, J. L. 2015. Alternativas de manejo sustentable de la subcuenca del río Pitura, Provincia de Imbabura, Ecuador. Master's thesis Universidad Nacional de La Plata. [en línea] disponible en: https://goo.gl/s6Bqmn.

Reynoldson, T. B y J. F. Wright. 2000. The reference condition: problems and solutions. Assessing the biological quality of fresh waters: RIVPACS and other techniques. Proceedings of an International Workshop held in Oxford, UK, on 16-18 September 1997. [en línea] disponible en: https://goo. gl/QCG36i chapter Assessing the biological quality of fresh waters: RIVPACS and other techniques. Proceedings of an International Workshop held in Oxford, UK, on 16-18 September 1997, pp. 293-309.

Rodríguez Quiñónez, D. E. 2012. “El desarrollo sustentable de la Cuenca Amazónica en la Agenda Ambiental de la Comunidad Andina (Tema Central)." Revista del Centro Andino de Estudios Internacionales 12:73-112. [en línea] disponible en : https://goo.gl/BGUzY2.

Roldan Perez, G. A. 1988. Guía para el estudio de macroinvertebrados acuáticos del departamento de Antio- quia. Universidad de Antioquía. [en línea] disponible en: https://goo.gl/BDp9AS.

Roldan Perez, G. A. 1996. Guía para el estudio de macroinvertebrados acuáticos del departamento de Antioquia. Fondo Para la Proteccioón del Medio Ambiente "Jose Celestino Mutis". [en línea] disponible en: https:/ /goo.gl/AetWa7.

Rosenberg, D y V. Resh. 1993. Freshwater biomonitoring and benthic macroinvertebrates. Springer US. [en línea] disponible en: https://goo.gl/E6czvb.

Solanes, M y H. Peña. 2003. La gobernabilidad de la gestión del agua en Ecuador. GWP - SAMTAC. [en línea] disponible en: https://goo.gl/itq1k9.

Sporka, F, H Vlek, E Bulánková y I. Krno. 2006. "Influence of seasonal variation on bioassessment of streams using macroinvertebrates." The Ecological Status of European Rivers: Evaluation and Intercalibration of Assessment Methods 188:543555. [en línea] doi: https://doi.org/10.1007/ 978--1--4020--5493--8_36.

Terneus, E. 2015. "Efectos del potencial represamiento de ríos sobre la ictiofauna: una aproximación al establecimiento de caudales ecológicos." Qualitas 10:64-84. [en línea] disponible en: https://goo.gl/eMWSZc.

Terneus, E, M Racines y K. Hernández. 2012. “Evaluación ecológica del río Lliquino a través de macroinvertebrados acuáticos, Pastaza, Ecuador." Universidad del Valle, Colombia: Facultad de Ciencias Naturales y Exactas 16:31-45. [en línea] disponible en: https://goo.gl/8vnhxN.

Yánez, P y M. Bárcenas. 2012. "Determinación de los niveles de tolerancia a hidrocarburos y potencial de fitorremediación de cuatro especies vegetales del sector Baeza-El Chaco, Ecuador." La Granja Revista de Ciencias de la Vida 15(1):2748. [en línea] doi: http://dx.doi.org/10.17163/ lgr.n15.2012.03. 